\title{
A Rare Case of Extra-Gonadal Oestrogen-Secreting Choriocarcinoma in a Male
}

\author{
Yuxuan Zhou \\ Department of General Medicine, Whangarei Hospital, Northland District Health Board, Whangarei, New Zealand
}

Received: 04/08/2021

Accepted: $13 / 08 / 2021$

Published: 03/09/2021

How to cite this article: Zhou Y. A rare case of extra-gonadal oestrogen-secreting choriocarcinoma in a male. EJCRIM 2021;8: doi:10.12890/2021_002814.

Conflicts of Interests: The authors declare there are no competing interests.

Patient Consent: Written informed consent to publish this case report was received from the: patient's next of kin. A copy of this consent is available on request.

This article is licensed under a Commons Attribution Non-Commercial 4.0 License

\section{ABSTRACT}

This report describes a rare case of an extra-gonadal oestrogen-secreting tumour in a male patient. An otherwise healthy 60 -year-old man presented to our hospital with a 3-month history of shortness of breath and weight loss. Blood panels and histology supported the diagnosis of an oestrogen-secreting choriocarcinoma. Unfortunately, the patient died soon after his diagnosis. The highlighting features of this case are: (1) the difficulty of confirming a diagnosis in a rapidly deteriorating patient; (2) the rarity of oestrogen-secreting extra-gonadal tumours in males; and (3) the aggressive rate of tumour progression seen on sequential imaging.

\section{LEARNING POINTS}

- Extra-gonadal oestrogen-secreting tumours in males are rare, but early empiric chemotherapy can be of benefit if this diagnosis is suspected.

- Tumour progression can be rapid, and so prompt referral to oncology services is critical for emergent management of the condition.

- Full endocrine blood panels including oestrogen, $\beta$ human chorionic gonadotrophin and $\alpha$ fetoprotein are useful to help diagnose the condition.

\section{KEYWORDS}

Oestrogen-secreting tumour in males, choriocarcinoma, tumour progression, extra-gonadal

\section{CASE DESCRIPTION}

A 60-year-old man, an active dairy farmer, presented to hospital with a 3-month history of worsening fatigue, shortness of breath, and a 10 kg weight loss. In addition, he had noted new and painless gynaecomastia over the previous 3 weeks, with significant loss of libido.

He initially presented to his general practitioner (GP) who performed a panel of endocrine blood tests. Of significance, he had elevated progesterone $97.8 \mathrm{nmol} / \mathrm{I}$ (normal <3.9), oestrodial $4111 \mathrm{pmol} / \mathrm{I}$ (normal <161), prolactin $635 \mathrm{mlU} / \mathrm{I}$ (normal <301), morning cortisol 819 $\mathrm{nmol} / \mathrm{I}$ (normal morning peak 170-500) and $\beta$ human chorionic gonadotrophin (HCG) >10,000 IU/l. In addition, levels of follicle-stimulating hormone $(\mathrm{FSH})<0.5 \mathrm{IU} / \mathrm{I}$ (normal 2-12), luteinising hormone (LH) $3.0 \mathrm{IU} / \mathrm{I}$ (normal 2-9) and morning adrenocorticotrophic hormone (ACTH) $3 \mathrm{pmol} / \mathrm{l}(2-11$ at $0900 \mathrm{~h}$ ) were also recorded. His thyroid function tests revealed severe hyperthyroidism, with a free T4 of $42 \mathrm{pmol} / \mathrm{l}$ (normal 12-22) and a TSH of $<0.01 \mathrm{mU} / \mathrm{I}$ (normal 0.27-4.2). Other blood tests including a fetoprotein were within normal limits. 
An outpatient chest $x$-ray revealed extensive bilateral cannonball metastases (Fig. 1). Following the chest x-ray, the patient was admitted to hospital acutely to the General Medicine department, where an urgent staging CT of the chest, abdomen and pelvis was performed, confirming the x-ray findings. It was also noted on physical examination that there were no palpable masses or tenderness in his testes.

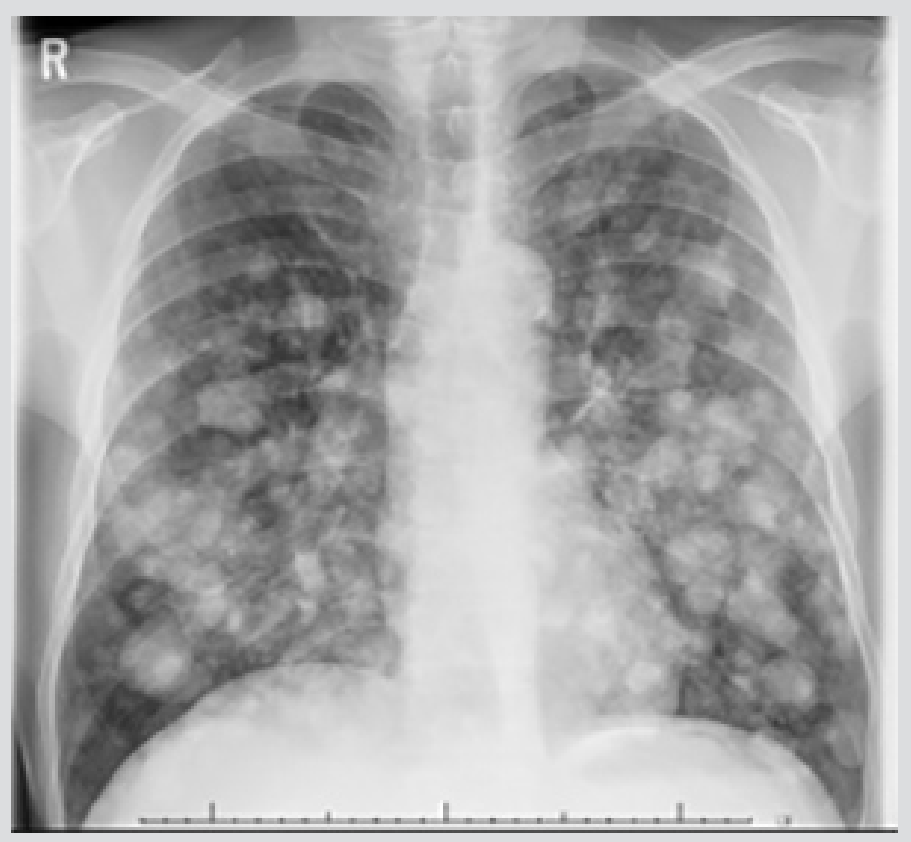

Figure 1. Chest $x$-ray on admission showing extensive cannonball pulmonary metastases

A lung biopsy was performed on day 4 of his admission. Unfortunately, this was complicated by a large right-sided pneumothorax which required urgent chest drain insertion due to rapid shortness of breath and desaturation. The chest drain relieved his symptoms temporarily, but over the next 5 days his breathlessness and tachypnoea worsened again.

On day 9 of his admission, he was noted to be tachypnoeic with a respiration rate of 45/min. A venous blood gas sample was taken, returning with a lactate of $12.2 \mathrm{mmol} / \mathrm{I}$ (normal <2). This was associated with a firm tender abdomen and 'egg yolk'-coloured diarrhoea. A CT pulmonary angiogram was then performed, which excluded pulmonary emboli and pneumothorax. However, it showed rapid progression of his pulmonary metastases with a significant increase in tumour size of between $10 \%$ and $20 \%$ over a 6-day period (Fig. 2). A CT mesenteric angiogram was also performed which excluded bowel ischaemia. However, the CT noted the known significant hepatomegaly down to the sacrum (Fig. 3). Unfortunately, following this rapid and acute deterioration, the patient died on day 10 of his admission.

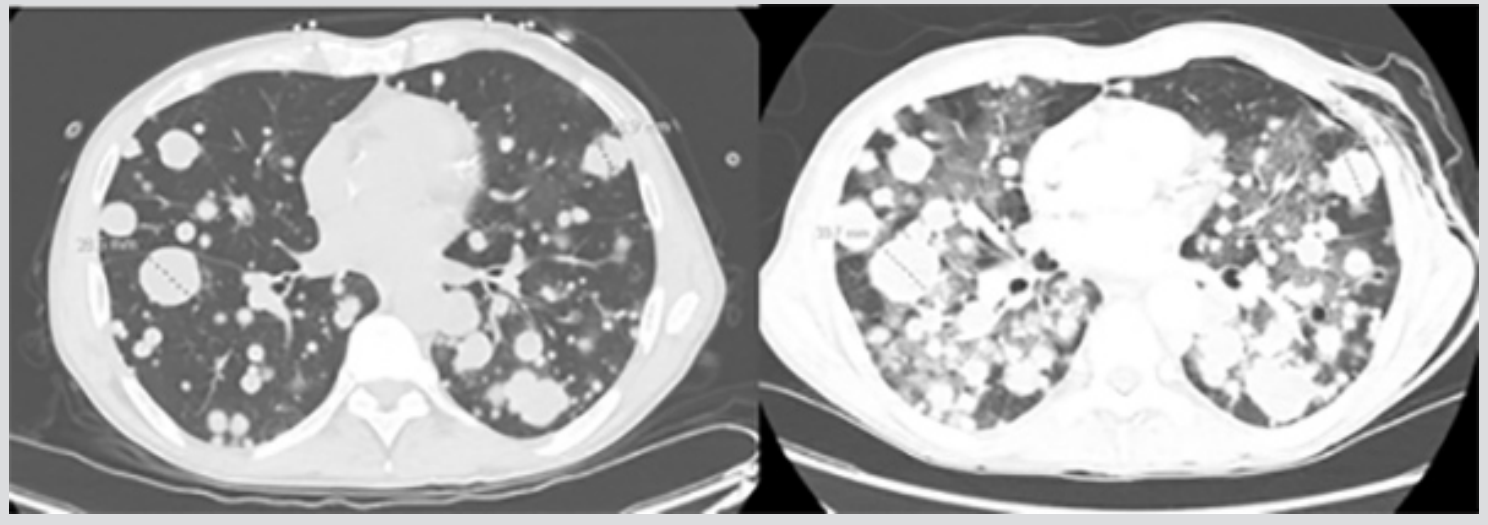

Figure 2. Comparison of CT of the chest images 6 days apart, showing rapid tumour progression; the left image is the earlier scan 
The histology results showed scarce viable tumour cells among large amounts of tumour necrosis (Fig. 4). Immunohistochemistry stains demonstrated strong positivity for CK7. PAX8, synaptophysin and melanin A were negative. GATA-3 and $\beta$-HCG staining were both positive. This supported the diagnosis of choriocarcinoma.

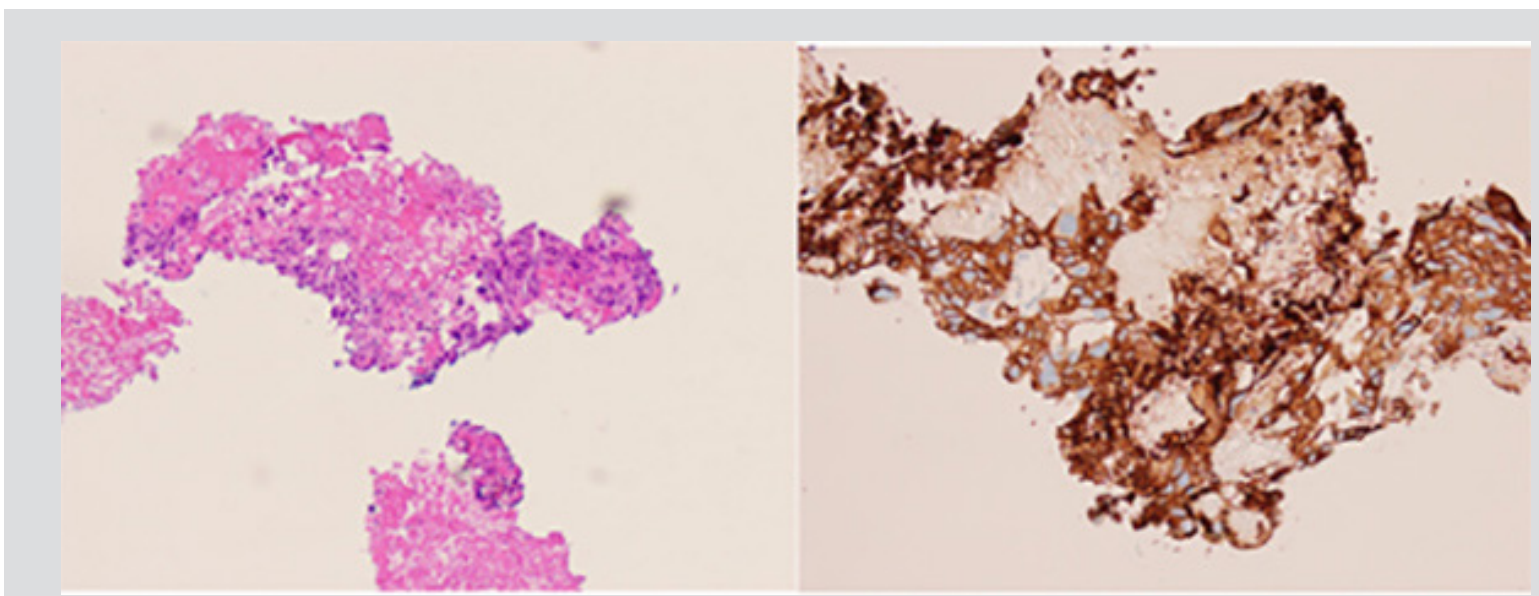

Figure 4. Histology images of the lung biopsy tissue with H\&E stain on the left and CK7 stain (strongly positive) on the right

\section{DISCUSSION}

Non-ovarian oestrogen-producing tumours are rare, with only a small number of cases being described in the literature ${ }^{[1]}$. In males, the most likely diagnosis is choriocarcinoma ${ }^{[2]}$. Evidence to support this diagnosis includes hyper-oestrogenic symptoms, raised $\beta$-HCG levels and normal a fetoprotein levels.

Although choriocarcinoma is within the umbrella of germ cell tumours, the primary site of malignancy is not always the sex organs ${ }^{[3]}$. A number of cases in the literature have described extra-gonadal primary choriocarcinoma, with the lung, mediastinum and the intestinal tract the most common sites ${ }^{[4]}$. In our patient, physical examination of the testes was unremarkable and CT images that captured the testes did not show any significant abnormality. 
Of particular note, the growth rate of this tumour was exceptionally aggressive. Review of the sequential CT scans that were taken only 6 days apart revealed isolated lesions in the lung that grew by up to $20 \%$ within that short time period (doubling time of approximately 21 days). There are currently no published data quantifying tumour growth rates in choriocarcinoma.

The complexity of this patient's oncological issue with his acute deterioration following biopsy was difficult to manage.In retrospect, earlier referral to the Oncology service may have been of benefit as choriocarcinoma are generally responsive to chemotherapy. In this particular situation, the patient could have been managed from the Oncology service without histology, and started on empiric chemotherapy. However, the patient's acute deterioration with regards to his pneumothorax and lactic acidosis would have resulted in a guarded prognosis despite appropriate chemotherapy.

In summary, this is a rare and unusual case of an aggressive extra-gonadal oestrogen-producing choriocarcinoma in a male patient. If tissue diagnosis cannot be confirmed due to an insufficient tissue sample, then the differential diagnosis should include paraneoplastic syndrome from a non-germ cell tumour, Leydig cell tumour, as well as feminising adrenocortical tumours. Other considerations in such a presentation include early investigation for multiple endocrine abnormalities.

\section{REFERENCES}

Fancellu A, Pinna A, Porcu A. Feminizing adrenocortical carcinoma with distant metastases: can surgery be considered? Clin Pract 2014;4(2):651.

Oosterhuis JW, Looijenga LHJ. Testicular germ-cell tumours in a broader perspective. Nat Rev Cancer 2005;5(3):210-222.

Holt LP, Melcher DH, Colquhoun J. Extra-gonadal choriocarcinoma in the male. Postgrad Med J 1965;41:134-138.

Fine G, Smith RW Jr, Pachter MR. Primary extragenital choriocarcinoma in the male subject. Case report and review of the literature. Am J Med 1962;32:776-794. 